\title{
COALGEBRA-GALOIS EXTENSIONS FROM THE EXTENSION THEORY POINT OF VIEW
}

\author{
TOMASZ BRZEZIŃSKI
}

\begin{abstract}
Coalgebra-Galois extensions generalise Hopf-Galois extensions, which can be viewed as non-commutative torsors. In this paper it is analysed when a coalgebra-Galois extension is a separable, split, or strongly separable extension.
\end{abstract}

\section{INTRODUCTION}

Given a coalgebra $C$, an algebra $A$ and a right coaction $\rho^{A}: A \rightarrow A \otimes C$ one can define a fixed point subalgebra $B$ of $A$ as consisting of all those elements of $A$ over which the coaction is left-linear. In this way one obtains an extension $B \hookrightarrow A$, which is called a coalgebra-Galois extension if a certain canonical left $A$-module, right $C$-comodule map is bijective 国 [3]. The aim of this article is to analyse such coalgebra-Galois extensions from the extension theory point of view. In particular we study the problem when such extensions are separable, split or strongly separable extensions. This problem is put in a broader context of entwining structures and entwined modules introduced in [四] [2], as a generalisation of a Doi-Hopf datum and Doi-Koppinen modules [10] [15, respectively. We make use of the notion of a separability of a functor introduced in [17], and, as a byproduct, we generalise some of the results of [5] obtained recently for Doi-Koppinen modules.

The paper is organised as follows. In Section 2 we recall definitions and give examples of entwining structures and entwined modules. In Section 3 we analyse when certain functors between categories of entwined modules induced by morphisms of entwining structures are separable. In Section 4 we apply the results of Section 3 to prove that a sufficient and necessary condition for a coalgebra-Galois extension to be separable is the separability of a certain induction functor. This, in turn, is equivalent to the existence of a normalised integral in the canonical entwining structure. In Section 5 we analyse when a coalgebra-Galois extension is a split extension. This turns out to be related to the separability of the forgetful functor from the category of entwined modules to the category of right modules - another special case of the main theorem in Section 3. Finally, in Section 6 we study the problem when a coalgebra-Galois extension is a strongly separable extension in the sense of [14].

We work over a commutative ring $k$ with identity 1 . We assume that all the algebras are over $k$, associative and unital, and the coalgebras are over $k$, coassociative and counital. Unadorned tensor product is over $k$. For any $k$-modules $V, W$ the symbol $\operatorname{Hom}(V, W)$ denotes the $k$-module of $k$-linear maps $V \rightarrow W$ and the identity map $V \rightarrow V$ is denoted by $V$. The twist map between $k$-modules $V, W$ is denoted

1991 Mathematics Subject Classification. 16W30, $17 \mathrm{~B} 37$.

Lloyd's of London Tercentenary Fellow.

On leave from: Department of Theoretical Physics, University of Lódź, Pomorska 149/153, 90-236, Łódź, Poland. 
by twist : $V \otimes W \rightarrow W \otimes V, v \otimes w \mapsto w \otimes v$. We also implicitly identify $V$ with $V \otimes k$ and $k \otimes V$ via the canonical isomorphisms.

For a $k$-algebra $A$ we use $\mu_{A}$ to denote the product as a map and $1_{A}$ to denote the identity both as an element of $A$ and as a map $k \rightarrow A, \alpha \mapsto \alpha 1_{A} \cdot \mathbf{M}_{A}$ (resp. ${ }_{A} \mathbf{M}$ ) denotes the category of right (resp. left) $A$-modules. The morphisms in this category are denoted by $\operatorname{Hom}_{A}(M, N)$ (resp. $\left.{ }_{A} \operatorname{Hom}(M, N)\right)$. For any $M \in \mathbf{M}_{A}$ (resp. $M \in{ }_{A} \mathbf{M}$ ), the symbol $\rho_{M}$ (resp. ${ }_{M} \rho$ ) denotes the action as a map (on elements the action is denoted by a dot). We often write $M_{A}$ (resp. $\left.{ }_{A} M\right)$ to indicate in which context the $A$-module $M$ appears. For any $M \in \mathbf{M}^{A}, N \in{ }^{A} \mathbf{M}$ we will write $\mathrm{eq}_{M_{A} N}: M \otimes A \otimes N \rightarrow M \otimes N$ for the action equalising map defining tensor product $M \otimes_{A} N$, i.e., $\mathrm{eq}_{M_{A} N}=\rho_{M} \otimes N-M \otimes_{N} \rho, M \otimes_{A} N=\operatorname{coker}\left(\mathrm{eq}_{M_{A} N}\right)$.

For a $k$-coalgebra $C$ we use $\Delta_{C}$ to denote the coproduct and $\epsilon_{C}$ to denote the counit. Notation for comodules is similar to that for modules but with subscripts replaced by superscripts, i.e. $\mathbf{M}^{C}$ is the category of right $C$-comodules, $\rho^{M}$ is a right coaction etc. We use the Sweedler notation for coproducts and coactions, i.e. $\Delta_{C}(c)=c_{(1)} \otimes c_{(2)}, \rho^{M}(m)=m_{(0)} \otimes m_{(1)}$ (summation understood). For any $V \in \mathbf{M}^{C}, W \in{ }^{C} \mathbf{M}, V \square_{C} W$ denotes the cotensor product, which is defined by the exact sequence

$$
0 \longrightarrow V \square_{C} W \longrightarrow V \otimes W \stackrel{\mathrm{eq}^{V^{C} W}}{\longrightarrow} V \otimes C \otimes W
$$

where $\mathrm{eq}^{V^{C} W}$ is the coaction equalising map, i.e., $\mathrm{eq}^{V^{C} W}=\rho^{V} \otimes W-V \otimes{ }^{W}$.

\section{Preliminaries on entwining Structures AND COALGebra-Galois EXTENSIONS}

Definition 2.1. An entwining structure (over $k$ ) is a triple $(A, C)_{\psi}$ consisting of a $k$-algebra $A$, a $k$-coalgebra $C$ and a $k$-linear map $\psi: C \otimes A \rightarrow A \otimes C$ satisfying

$$
\begin{aligned}
& \psi \circ\left(C \otimes \mu_{A}\right)=\left(\mu_{A} \otimes C\right) \circ(A \otimes \psi) \circ(\psi \otimes A), \quad \psi \circ\left(C \otimes 1_{A}\right)=1_{A} \otimes C, \\
& \left(A \otimes \Delta_{C}\right) \circ \psi=(\psi \otimes C) \circ(C \otimes \psi) \circ\left(\Delta_{C} \otimes A\right), \quad\left(A \otimes \epsilon_{C}\right) \circ \psi=\epsilon_{C} \otimes A .
\end{aligned}
$$

A morphism of entwining structures is a pair $(f, g):(A, C)_{\psi} \rightarrow(\tilde{A}, \tilde{C})_{\tilde{\psi}}$, where $f: A \rightarrow \tilde{A}$ is an algebra map, $g: C \rightarrow \tilde{C}$ is a coalgebra map, and $(f \otimes g) \circ \psi=$ $\tilde{\psi} \circ(g \otimes f)$.

The category of entwining structures is a tensor category with tensor product $(A, C)_{\psi} \otimes(\tilde{A}, \tilde{C})_{\tilde{\psi}}=(A \otimes \tilde{A}, C \otimes \tilde{C})_{(A \otimes \text { twist } \otimes \tilde{C}) \circ(\psi \otimes \tilde{\psi}) \circ(C \otimes \text { twist } \otimes \tilde{A})}$, and unit object $(k, k)_{\text {twist }}$.

For $(A, C)_{\psi}$ we use the notation $\psi(c \otimes a)=a_{\alpha} \otimes c^{\alpha}$ (summation over a Greek index understood), for all $a \in A, c \in C$. The notion of an entwining structure was introduced in [4, Definition 2.1]. It is self-dual in the sense that conditions in Definition 2.1 are invariant under the operation consisting of interchanging of $A$ with $C, \mu_{A}$ with $\Delta_{C}$, and $1_{A}$ with $\epsilon_{C}$, and reversing the order of maps. Below are two classes of examples of entwining structures coming from Galois-extensions.

Example 2.2 ([3]). Let $C$ be a coalgebra, $A$ an algebra and a right $C$-comodule. Let $B:=\left\{b \in A \mid \rho^{A}(b a)=b \rho^{A}(a)\right\}$ and assume that the canonical left $A$-module, right $C$-comodule map can $: A \otimes_{B} A \rightarrow A \otimes C, a \otimes a^{\prime} \mapsto a \rho^{A}\left(a^{\prime}\right)$, is bijective. Let $\psi: C \otimes A \rightarrow A \otimes C$ be a $k$-linear map given by $\psi(c \otimes a)=\operatorname{can}\left(\operatorname{can}^{-1}\left(1_{A} \otimes c\right) a\right)$. 
Then $(A, C)_{\psi}$ is an entwining structure. The extension $B \hookrightarrow A$ is called a coalgebraGalois extension (or a $C$-Galois extension) and is denoted by $A(B)^{C} \cdot(A, C)_{\psi}$ is the canonical entwining structure associated to $A(B)^{C}$. A coalgebra-Galois extension $A(B)^{C}$ is said to be copointed if there exists a group-like $e \in C$ such that $\rho^{A}\left(1_{A}\right)=$ $1_{A} \otimes e$.

Dually we have

Example 2.3 ([3]). Let $A$ be an algebra, $C$ a coalgebra and a right $A$-module. Let $B:=C / I$, where $I$ is a coideal in $C$,

$$
I:=\operatorname{span}\left\{(c \cdot a)_{(1)} \xi\left((c \cdot a)_{(2)}\right)-c_{(1)} \xi\left(c_{(2)} \cdot a\right) \mid a \in A, c \in C, \xi \in C^{*}\right\},
$$

and assume that the canonical left $C$-comodule, right $A$-module map cocan : $C \otimes$ $A \rightarrow C \square_{B} C, c \otimes a \mapsto c_{(1)} \otimes c_{(2)} \cdot a$, is bijective. Let $\psi: C \otimes A \rightarrow A \otimes C$ be a $k$-linear map given by $\psi=\left(\epsilon_{C} \otimes A \otimes C\right) \circ\left(\operatorname{cocan}^{-1} \otimes C\right) \circ\left(C \otimes \Delta_{C}\right) \circ$ cocan. Then $(A, C)_{\psi}$ is an entwining structure. The coextension $C \rightarrow B$ is called an algebra-Galois coextension (or an $A$-Galois coextension) and is denoted by $C(B)_{A} \cdot(A, C)_{\psi}$ is the canonical entwining structure associated to $C(B)_{A}$. An algebra-Galois coextension $C(B)_{A}$ is said to be pointed if there exists an algebra map $\kappa: A \rightarrow k$ such that $\epsilon_{C} \circ \rho_{C}=\epsilon_{C} \otimes \kappa$.

Associated to an entwining structure is the category of entwined modules.

Definition 2.4. Let $(A, C)_{\psi}$ be an entwining structure. An (entwined) $(A, C)_{\psi^{-}}$ module is a right $A$-module, right $C$-comodule $M$ such that

$$
\rho^{M} \circ \rho_{M}=\left(\rho_{M} \otimes C\right) \circ(M \otimes \psi) \circ\left(\rho^{M} \otimes A\right),
$$

(explicitly: $\left.\rho^{M}(m \cdot a)=m_{(0)} \cdot a_{\alpha} \otimes m_{(1)}^{\alpha}, \forall a \in A, m \in M\right)$. A morphism of $(A, C)_{\psi^{-}}$ modules is a right $A$-module map which is also a right $C$-comodule map. The category of $(A, C)_{\psi}$-modules is denoted by $\mathbf{M}_{A}^{C}(\psi)$.

The category $\mathbf{M}_{A}^{C}(\psi)$ was introduced and studied in [2]. An example of such modules are Doi-Koppinen modules introduced in [10], [15]. In this paper we will be concerned with two covariant functors between categories of entwined modules, which are special cases of the construction in [2, Section 3] (see also [0] for the Doi-Koppinen case). These functors are induced by certain morphisms of entwining structures.

Definition 2.5. Let $(f, g):(A, C)_{\psi} \rightarrow(\tilde{A}, \tilde{C})_{\tilde{\psi}}$ be a morphism of entwining structures. View $C$ as a left $\tilde{C}$-comodule via ${ }^{C} \rho=(g \otimes C) \circ \Delta_{C}$ and $C \otimes \tilde{A}$ as a right $\tilde{C}$-comodule via $\rho^{C \otimes \tilde{A}}=(C \otimes \tilde{\psi}) \circ(C \otimes g \otimes \tilde{A}) \circ\left(\Delta_{C} \otimes \tilde{A}\right)$. Then $(f, g)$ is said to be an admissible morphism iff:

(i) for all $\tilde{M} \in \mathbf{M}_{\tilde{A}}^{\tilde{C}}(\tilde{\psi}), \tilde{M} \square_{\tilde{C}}(C \otimes C)=\left(\tilde{M} \square_{\tilde{C}} C\right) \otimes C$,

(ii) for all $M \in \mathbf{M}_{A},(M \otimes(C \otimes \tilde{A})) \square_{\tilde{C}} C=M \otimes\left((C \otimes \tilde{A}) \square_{\tilde{C}} C\right)$.

For example, if $C, \tilde{C}$ are $k$-flat then $(f, g)$ is an admissible morphism provided that $\tilde{C}_{C}$ is coflat. On the other hand if $k$ is a regular ring or a field every morphism is admissible. Also, it can be easily checked that the following morphisms $\left(A, \epsilon_{C}\right)$ : $(A, C)_{\psi} \rightarrow(A, k)_{\text {twist }}$ and $\left(1_{A}, C\right):(k, C)_{\text {twist }} \rightarrow(A, C)_{\psi}$ are admissible.

\footnotetext{
${ }^{1}$ Although the paper [2] is restricted to $k$ being a field, all the results of [2] quoted in the present paper can easily be seen to hold for a general $k$.
} 
Example 2.6. Let $(f, g):(A, C)_{\psi} \rightarrow(\tilde{A}, \tilde{C})_{\tilde{\psi}}$ be an admissible morphism of entwining structures. View $\tilde{A}$ as a right $A$-module via $\rho_{\tilde{A}}=\mu_{\tilde{A}} \circ(\tilde{A} \otimes f)$, and $C$ as a right $\tilde{C}$-comodule via $\rho^{C}=(C \otimes g) \circ \Delta_{C}$. Then:

(1) For any $\tilde{M} \in \mathbf{M}_{\tilde{A}}^{\tilde{C}}(\tilde{\psi}), \tilde{M} \square_{\tilde{C}} C$ is an $(A, C)_{\psi}$-module with structure maps $\rho^{\tilde{M}} \square_{\tilde{C}} C=\tilde{M} \otimes \Delta_{C}$ and

$$
\rho_{\tilde{M} \square_{\tilde{C} C}}: \tilde{M} \square_{\tilde{C}} C \otimes A \rightarrow \tilde{M} \square_{\tilde{C}} C, \quad \sum_{i} \tilde{m}_{i} \otimes c_{i} \otimes a=\sum_{i} \tilde{m}_{i} f\left(a_{\alpha}\right) \otimes c_{i}^{\alpha} .
$$

(2) For any $M \in \mathbf{M}_{A}^{C}(\psi), M \otimes_{A} \tilde{A}$ is an $(\tilde{A}, \tilde{C})_{\tilde{\psi}}$-module with structure maps $\rho_{M \otimes_{A} \tilde{A}}=M \otimes_{A} \mu_{\tilde{A}}$ and

$$
\rho^{M \otimes_{A} \tilde{A}}: M \otimes_{A} \tilde{A} \rightarrow M \otimes_{A} \tilde{A} \otimes \tilde{C}, \quad m \otimes \tilde{a} \mapsto m_{(0)} \otimes \tilde{a}_{\alpha} \otimes g\left(m_{(1)}\right)^{\alpha} .
$$

(3) The covariant functor $-\square_{\tilde{C}} C: \mathbf{M}_{\tilde{A}}^{\tilde{C}}(\tilde{\psi}) \rightarrow \mathbf{M}_{A}^{C}(\psi)$ is the right adjoint of $-\otimes_{A} \tilde{A}: \mathbf{M}_{A}^{C}(\psi) \rightarrow \mathbf{M}_{\tilde{A}}^{\tilde{C}}(\tilde{\psi})$. The adjunctions are:

$$
\forall M \in \mathbf{M}_{A}^{C}(\psi), \quad \Phi_{M}: M \rightarrow\left(M \otimes_{A} \tilde{A}\right) \square_{\tilde{C}} C, \quad m \mapsto m_{(0)} \otimes 1_{\tilde{A}} \otimes m_{(1)},
$$

$\forall \tilde{M} \in \mathbf{M}_{\tilde{A}}^{\tilde{C}}(\tilde{\psi}), \quad \Psi_{\tilde{M}}:\left(\tilde{M} \square_{\tilde{C}} C\right) \otimes_{A} \tilde{A} \rightarrow \tilde{M}, \quad \sum_{i} \tilde{m}_{i} \otimes c_{i} \otimes \tilde{a} \mapsto \sum_{i} \tilde{m}_{i} \cdot \tilde{a} \epsilon_{C}\left(c_{i}\right)$.

Applying Example 2.6 to morphisms $\left(A, \epsilon_{C}\right):(A, C)_{\psi} \rightarrow(A, k)_{\mathrm{twist}}$ and $\left(1_{A}, C\right)$ : $(k, C)_{\text {twist }} \rightarrow(A, C)_{\psi}$ one obtains

Example 2.7. Let $(A, C)_{\psi}$ be an entwining structure. Then

(1) If $M$ is a right $A$-module then $M \otimes C$ is an $(A, C)_{\psi}$-module with the coaction $M \otimes \Delta_{C}$ and the action $(m \otimes c) \cdot a=m \cdot \psi(c \otimes a)$, for all $a \in A, c \in C$ and $m \in M$. In particular $A \otimes C \in \mathbf{M}_{A}^{C}(\psi)$. The operation $M \mapsto M \otimes C$ defines a covariant functor $-\otimes C: \mathbf{M}_{A} \rightarrow \mathbf{M}_{A}^{C}(\psi)$ which is the right adjoint of the forgetful functor $\mathbf{M}_{A}^{C}(\psi) \rightarrow \mathbf{M}_{A}$.

(2) If $V$ is a right $C$-comodule then $V \otimes A \in \mathbf{M}_{A}^{C}(\psi)$ with the action $V \otimes \mu_{A}$ and the coaction $v \otimes a \mapsto v_{(0)} \otimes \psi\left(v_{(1)} \otimes a\right)$ for any $a \in A$ and $v \in V$. In particular $C \otimes A \in \mathbf{M}_{A}^{C}(\psi)$. The operation $V \mapsto V \otimes A$ defines a covariant functor $-\otimes C$ : $\mathbf{M}^{C} \rightarrow \mathbf{M}_{A}^{C}(\psi)$, which is the left adjoint of the forgetful functor $\mathbf{M}_{A}^{C}(\psi) \rightarrow \mathbf{M}^{C}$.

Another class of examples of entwined modules comes from (co)algebra-Galois (co)extensions [3]

\section{Example 2.8.}

(1) Let $(A, C)_{\psi}$ be the canonical entwining structure associated to a coalgebraGalois extension $A(B)^{C}$. Then $A$ is an $(A, C)_{\psi}$-module via $\rho^{A}$ and $\mu_{A}$.

(2) Let $(A, C)_{\psi}$ be the canonical entwining structure associated to an algebraGalois coextension $C(B)_{A}$. Then $C$ is an $(A, C)_{\psi}$-module via $\rho_{C}$ and $\Delta_{C}$.

\section{Separable functors of entwined modules}

In this section we analyse when functors described in Example 2.6 are separable. Recall from [17] that a covariant functor $F: \mathcal{C} \rightarrow \mathcal{D}$ is separable if the natural transformation $\operatorname{Hom}_{\mathcal{C}}(-,-) \rightarrow \operatorname{Hom}_{\mathcal{D}}(F(-), F(-))$ splits. In this paper we are dealing with the pairs of adjoint functors, so that the following characterisation of separable functors, obtained in [20] [8], is of great importance 
Theorem 3.1. Let $G: \mathcal{D} \rightarrow \mathcal{C}$ be the right adjoint of $F: \mathcal{C} \rightarrow \mathcal{D}$ with adjunctions $\Phi: 1_{\mathcal{C}} \rightarrow G F$ and $\Psi: F G \rightarrow 1_{\mathcal{D}}$. Then

(1) $F$ is separable if and only if $\Phi$ splits, i.e., for all objects $C \in \mathcal{C}$ there exists a morphism $\nu_{C} \in \operatorname{Mor}_{\mathcal{C}}(G F(C), C)$ such that $\nu_{C} \circ \Phi_{C}=C$ and for all $f \in \operatorname{Mor}_{\mathcal{C}}(C, \tilde{C})$, $\nu_{\tilde{C}} \circ G F(f)=f \circ \nu_{C}$.

(2) $G$ is separable if and only if $\Psi$ cosplits, i.e., for all objects $D \in \mathcal{D}$ there exists a morphism $\nu_{D} \in \operatorname{Mor}_{\mathcal{D}}(D, F G(D))$ such that $\Psi_{D} \circ \nu_{D}=D$ and for all $f \in \operatorname{Mor}_{\mathcal{D}}(D, \tilde{D}), \nu_{\tilde{D}} \circ f=F G(f) \circ \nu_{D}$.

Definition 3.2. An admissible morphism $(f, g):(A, C)_{\psi} \rightarrow(\tilde{A}, \tilde{C})_{\tilde{\psi}}$ of entwining structures is said to be:

(1) integrable if there exists $\lambda \in \operatorname{Hom}_{A}\left((C \otimes \tilde{A}) \square_{\tilde{C}} C, A\right)$ such that the following diagrams commute

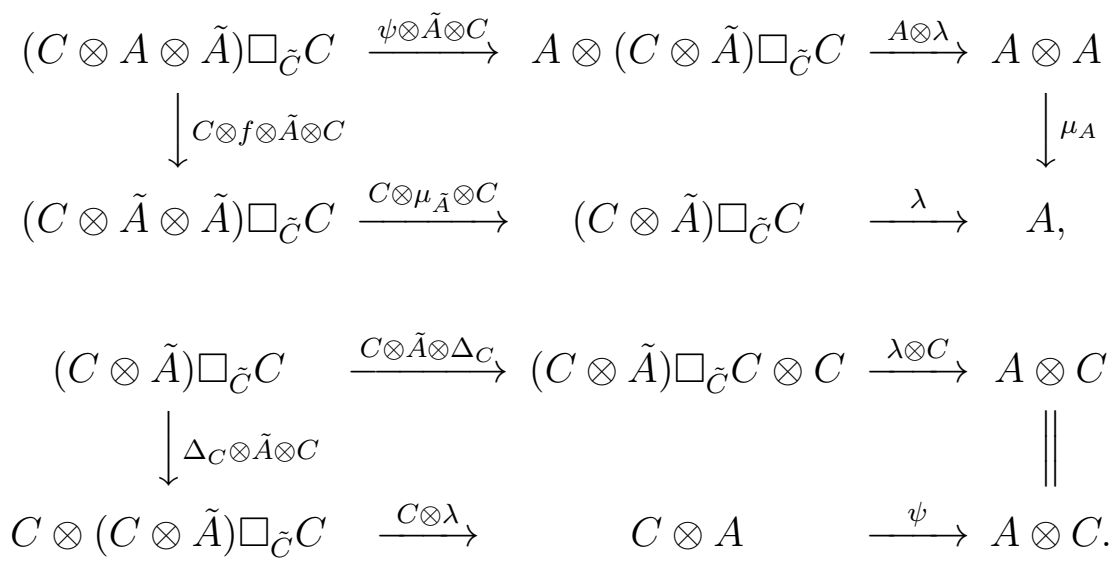

The right $A$-module structure of $(C \otimes \tilde{A}) \square_{\tilde{C}} C$ is as in Example 2.6(1), explicitly $\rho_{(C \otimes \tilde{A}) \square_{\tilde{C}} C}: c^{\prime} \otimes \tilde{a} \otimes c \otimes a \mapsto c^{\prime} \otimes \tilde{a} f\left(a_{\alpha}\right) \otimes c^{\alpha}$.

(2) totally integrable, if there exists $\lambda \in \operatorname{Hom}_{A}\left((C \otimes \tilde{A}) \square_{\tilde{C}} C, A\right)$ making it an integrable morphism and such that the following diagram

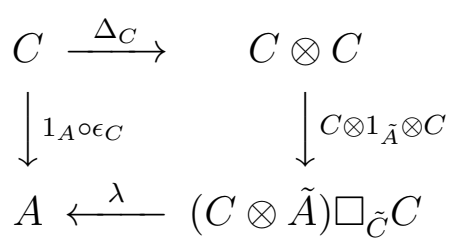

commutes.

Notice that the condition (3) makes sense because $\psi$ is a morphism in $\mathbf{M}_{A}^{C}(\psi)$, $(f, g)$ is admissible and $\left(C \otimes \mu_{\tilde{A}}\right) \circ(C \otimes f \otimes \tilde{A}): C \otimes A \otimes \tilde{A} \rightarrow C \otimes \tilde{A}$ is a left $\tilde{C}$-comodule map, where the $k$-modules involved are left $\tilde{C}$-comodules via $(g \otimes C) \circ \Delta_{C} \otimes A \otimes \tilde{A}$ and $(g \otimes C) \circ \Delta_{C} \otimes \tilde{A}$, respectively. Similarly, condition (耳) makes sense because $\Delta_{C}$ is a left $\tilde{C}$-comodule map and $\Delta_{C} \otimes \tilde{A}$ is a morphism in $\mathbf{M}_{\tilde{A}}^{\tilde{C}}(\tilde{\psi})$. Dually to Definition 3.2 one considers

Definition 3.3. An admissible morphism $(f, g):(A, C)_{\psi} \rightarrow(\tilde{A}, \tilde{C})_{\tilde{\psi}}$ of entwining structures is said to be: 
(1) cointegrable if there exists $\mathfrak{z} \in \operatorname{Hom}^{\tilde{C}}\left(\tilde{C},(\tilde{A} \otimes C) \otimes_{A} \tilde{A}\right)$ such that the following diagrams commute

$$
\begin{aligned}
& \tilde{C} \stackrel{\mathfrak{z}}{\longrightarrow} \quad(\tilde{A} \otimes C) \otimes_{A} \tilde{A} \quad \stackrel{\left(\tilde{A} \otimes \Delta_{C}\right) \otimes_{A} \tilde{A}}{\longrightarrow}(\tilde{A} \otimes C \otimes C) \otimes_{A} \tilde{A} \\
& \downarrow \Delta_{\tilde{C}} \quad \downarrow(\tilde{A} \otimes g \otimes C) \otimes_{A} \tilde{A} \\
& \tilde{C} \otimes \tilde{C} \stackrel{\tilde{C} \otimes \mathfrak{z}}{\longrightarrow} \tilde{C} \otimes(\tilde{A} \otimes C) \otimes_{A} \tilde{A} \quad \stackrel{\tilde{\psi} \otimes \tilde{C} \otimes \tilde{A}}{\longrightarrow} \quad(\tilde{A} \otimes \tilde{C} \otimes C) \otimes_{A} \tilde{A} \\
& \tilde{C} \otimes \tilde{A} \stackrel{\tilde{\psi}}{\longrightarrow} \quad \tilde{A} \otimes \tilde{C} \quad \stackrel{\tilde{A} \otimes \mathfrak{z}}{\longrightarrow} \quad(\tilde{A} \otimes \tilde{A} \otimes C) \otimes_{A} \tilde{A} \\
& \| \downarrow \downarrow\left(\mu_{\tilde{A}} \otimes C\right) \otimes_{A} \tilde{A} \\
& \tilde{C} \otimes \tilde{A} \stackrel{\mathfrak{z} \otimes \tilde{A}}{\longrightarrow}(\tilde{A} \otimes C) \otimes_{A} \tilde{A} \otimes \tilde{A} \stackrel{(\tilde{A} \otimes C)_{A} \otimes \mu_{\tilde{A}}}{\longrightarrow} \quad(\tilde{A} \otimes C) \otimes_{A} \tilde{A} .
\end{aligned}
$$

The right $\tilde{C}$-comodule structure of $(\tilde{A} \otimes C) \otimes_{A} \tilde{A}$ is as in Example 2.6(2), explicitly $\rho^{(\tilde{A} \otimes C) \otimes_{A} \tilde{A}}: \tilde{a} \otimes c \otimes \tilde{a}^{\prime} \mapsto \tilde{a} \otimes c_{(1)} \otimes \tilde{a}_{\alpha}^{\prime} \otimes g\left(c_{(2)}\right)^{\alpha}$.

(2) totally cointegrable, if there exists $\mathfrak{z} \in \operatorname{Hom}^{\tilde{C}}\left(\tilde{C},(\tilde{A} \otimes C) \otimes_{A} \tilde{A}\right)$ making it a cointegrable morphism and such that the following diagram

$$
\begin{aligned}
& \tilde{C} \stackrel{\mathfrak{z}}{\longrightarrow}(\tilde{A} \otimes C) \otimes_{A} \tilde{A} \\
& \downarrow 1_{\tilde{A}^{\circ} \epsilon_{\tilde{C}}} \\
& \left.\tilde{A} \stackrel{\mu_{\tilde{A} A}}{\longleftarrow} \tilde{A} \otimes_{A} \tilde{A} \otimes \epsilon_{C}\right) \otimes_{A} \tilde{A}
\end{aligned}
$$

commutes. Here $\mu_{\tilde{A}, A}: \tilde{A} \otimes_{A} \tilde{A} \rightarrow \tilde{A}$ is the natural map induced by $\mu_{\tilde{A}}$.

The right actions of $A$ on the $k$-modules involved in the above definition are as follows. For any $a \in A, \tilde{a}, \tilde{a}^{\prime} \in \tilde{A}, c, c^{\prime} \in C, \tilde{c} \in \tilde{C}:(\tilde{a} \otimes c) \cdot a=\tilde{a} f\left(a_{\alpha}\right) \otimes c^{\alpha}$, $\left(\tilde{a} \otimes c \otimes c^{\prime}\right)=\tilde{a} f\left(a_{\alpha \beta}\right) \otimes c^{\beta} \otimes c^{\prime \alpha},(\tilde{a} \otimes \tilde{c} \otimes c) \cdot a=\tilde{a} f\left(a_{\alpha}\right)_{\beta} \otimes \tilde{c}^{\beta} \otimes c^{\alpha},(\tilde{c} \otimes \tilde{a} \otimes c) \cdot a=$ $\tilde{c} \otimes \tilde{a} f\left(a_{\alpha}\right) \otimes c^{\alpha},\left(\tilde{a} \otimes \tilde{a}^{\prime} \otimes c\right) \cdot a=\tilde{a} \otimes \tilde{a}^{\prime} f\left(a_{\alpha}\right) \otimes c^{\alpha}$. Using properties of entwining structures and the fact that $(f, g)$ is a morphism of entwining structures one can easily convince oneself that all the maps featuring in Definition 3.3 are well-defined.

With these definitions at hand we can now state the main result of this section.

Theorem 3.4. Let $(f, g):(A, C)_{\psi} \rightarrow(\tilde{A}, \tilde{C})_{\tilde{\psi}}$ be an admissible morphism of entwining structures.

(1) If for all $M \in \mathbf{M}_{A}^{C}(\psi),\left(M \otimes_{A} \tilde{A}\right) \square_{\tilde{C}} C \subseteq \operatorname{coker}\left(\operatorname{eq}_{M_{A} \tilde{A}} \square_{\tilde{C}} C\right)$, then the functor $-\otimes_{A} \tilde{A}: \mathbf{M}_{A}^{C}(\psi) \rightarrow \mathbf{M}_{\tilde{A}}^{\tilde{C}}(\tilde{\psi})$ is separable if and only if $(f, g)$ is totally integrable.

(2) If for all $\tilde{M} \in \mathbf{M}_{\tilde{A}}^{\tilde{C}}(\tilde{\psi}), \operatorname{ker}\left(\mathrm{eq}^{\tilde{M}^{\tilde{C}} C} \otimes_{A} \tilde{A}\right) \subseteq\left(\tilde{M} \square_{\tilde{C}} C\right) \otimes_{A} \tilde{A}$, then the functor $-\square_{\tilde{C}} C: \mathbf{M}_{\tilde{A}}^{\tilde{C}}(\tilde{\psi}) \rightarrow \mathbf{M}_{A}^{C}(\psi)$ is separable if and only if $(f, g)$ is totally cointegrable.

Proof. (1) Let $(f, g)$ be totally integrable and assume that $\lambda$ is as in Definition 3.2. For all $M \in \mathbf{M}_{A}^{C}(\psi)$ define

$$
\tilde{\nu}_{M}:(M \otimes \tilde{A}) \square_{\tilde{C}} C \rightarrow M, \quad \sum_{i} m_{i} \otimes \tilde{a}_{i} \otimes c_{i} \mapsto \sum_{i} m_{i(0)} \cdot \lambda\left(m_{i(1)} \otimes \tilde{a}_{i} \otimes c_{i}\right) .
$$

Notice that the map $\tilde{\nu}_{M}$ is well-defined since the fact that $(f, g)$ is admissible implies that for any $x \in(M \otimes \tilde{A}) \square_{\tilde{C}} C$, one has $\left.\left(\rho^{M} \otimes \tilde{A} \otimes C\right)(x) \in(M \otimes(C \otimes \tilde{A})) \square_{\tilde{C}} C\right)=$ 
$M \otimes\left((C \otimes \tilde{A}) \square_{\tilde{C}} C\right)$. Take any $x=\sum_{i} m_{i} \cdot a_{i} \otimes \tilde{a}_{i} \otimes c_{i} \in(M \otimes \tilde{A}) \square_{\tilde{C}} C$. Then

$$
\begin{array}{rlr}
\tilde{\nu}_{M}(x) & =\sum_{i}\left(m_{i} \cdot a_{i}\right)_{(0)} \cdot \lambda\left(\left(m_{i} \cdot a_{i}\right)_{(1)} \otimes \tilde{a}_{i} \otimes c_{i}\right) & \\
& =\sum_{i} m_{i(0)} \cdot a_{i \alpha} \lambda\left(m_{i(1)}^{\alpha} \otimes \tilde{a}_{i} \otimes c_{i}\right) \quad\left(M \in \mathbf{M}_{A}^{C}(\psi)\right) \\
& =\sum_{i} m_{i(0)} \cdot \lambda\left(m_{i(1)} \otimes f\left(a_{i}\right) \tilde{a}_{i} \otimes c_{i}\right) \quad(\text { by (3) }) \\
& =\tilde{\nu}_{M}\left(\sum_{i} m_{i} \otimes f\left(a_{i}\right) \tilde{a}_{i} \otimes c_{i}\right) .
\end{array}
$$

The above calculation means that $\operatorname{Im}\left(\mathrm{eq}_{M_{A} \tilde{A}} \square_{\tilde{C}} C\right) \subseteq$ ker $\tilde{\nu}_{M}$, and together with the assumption that $-\square_{\tilde{C}} C$ preserves the cokernel of the action equalising map $\mathrm{eq}_{M_{A} \tilde{A}}$ imply that one can define the map $\nu_{M}:\left(M \otimes_{A} \tilde{A}\right) \square_{\tilde{C}} C \rightarrow M$ by the diagram

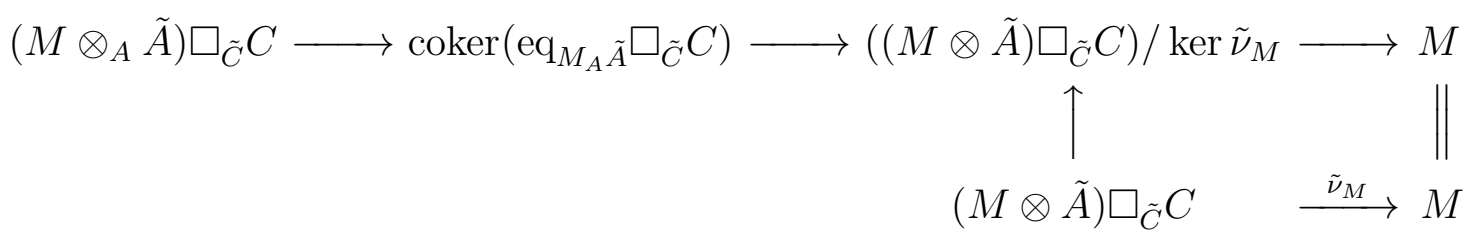

Slightly abusing the notation we will still write $\nu_{M}: \sum_{i} m_{i} \otimes \tilde{a}_{i} \otimes c_{i} \mapsto \sum_{i} m_{i(0)}$. $\lambda\left(m_{i(1)} \otimes \tilde{a}_{i} \otimes c_{i}\right)$.

To show that $\nu_{M}$ is a right $A$-module map, take any $a \in A$ and $x=\sum_{i} m_{i} \otimes \tilde{a}_{i} \otimes c_{i} \in$ $\left(M \otimes_{A} \tilde{A}\right) \square_{\tilde{C}} C$ and compute

$$
\begin{aligned}
\nu_{M}(x \cdot a) & =\nu_{M}\left(\sum_{i} m_{i} \otimes \tilde{a}_{i} f\left(a_{\alpha}\right) \otimes c_{i}^{\alpha}\right) \\
& =\sum_{i} m_{i(0)} \cdot \lambda\left(m_{i(1)} \otimes \tilde{a}_{i} f\left(a_{\alpha}\right) \otimes c_{i}^{\alpha}\right) \\
& =\sum_{i} m_{i(0)} \cdot \lambda\left(m_{i(1)} \otimes \tilde{a}_{i} \otimes c_{i}\right) a \quad\left(f \in \operatorname{Hom}_{A}\left((C \otimes \tilde{A}) \square_{\tilde{C}} C, A\right)\right) \\
& =\nu_{M}(x) \cdot a
\end{aligned}
$$

Furthermore we have

$$
\begin{aligned}
\nu_{M}\left(x_{(0)}\right) \otimes x_{(1)} & =\sum_{i} m_{i(0)} \cdot \lambda\left(m_{i(1)} \otimes \tilde{a}_{i} \otimes c_{i(1)}\right) \otimes c_{i(2)} \\
& =\sum_{i} m_{i(0)} \cdot \lambda\left(m_{i(2)} \otimes \tilde{a}_{i} \otimes c_{i}\right)_{\alpha} \otimes m_{i(1)}^{\alpha} \quad(\text { by (4) }) \\
& =\sum_{i} \rho^{M}\left(m_{i(0)} \cdot \lambda\left(m_{i(1)} \otimes \tilde{a}_{i} \otimes c_{i}\right)\right) \quad\left(M \in \mathbf{M}_{A}^{C}(\psi)\right) \\
& =\rho^{M} \circ \nu_{M}(x),
\end{aligned}
$$

which proves that $\nu_{M}$ is a right $C$-comodule map. Using (5) one easily finds that the adjunction $\Phi_{M}$ is splitted by $\nu_{M}$. It remains to be shown that $\nu_{M}$ is natural in 
$\mathbf{M}_{A}^{C}(\psi)$. Take any $M, N \in \mathbf{M}_{A}^{C}(\psi)$ and $\phi \in \operatorname{Hom}_{A}^{C}(M, N)$. Then

$$
\begin{aligned}
\nu_{N}\left(\sum_{i} \phi\left(m_{i}\right) \otimes \tilde{a}_{i} \otimes c_{i}\right) & =\sum_{i} \phi\left(m_{i}\right)_{(0)} \cdot \lambda\left(\phi\left(m_{i}\right)_{(1)} \otimes \tilde{a}_{i} \otimes c_{i}\right) \\
& =\sum_{i} \phi\left(m_{i(0)}\right) \cdot \lambda\left(m_{i(1)} \otimes \tilde{a}_{i} \otimes c_{i}\right) \\
& =\sum_{i} \phi\left(m_{i(0)} \cdot \lambda\left(m_{i(1)} \otimes \tilde{a}_{i} \otimes c_{i}\right)\right) \\
& =\phi \circ \nu_{M}(x),
\end{aligned}
$$

where we used that $\phi$ is a right $C$-comodule and right $A$-module map to derive the second and the third equalities respectively. This completes the proof that the functor $-\square_{\tilde{C}} C$ is separable.

Conversely, assume that $-\square_{\tilde{C}} C$ is separable and let $\nu_{M}$ be the corresponding splitting of $\Phi_{M}$. Define

$$
\lambda:(C \otimes \tilde{A}) \square_{\tilde{C}} C \rightarrow A, \quad \lambda=\left(A \otimes \epsilon_{C}\right) \circ \nu_{A \otimes C}\left(1_{A} \otimes(C \otimes \tilde{A}) \square_{\tilde{C}} C\right) .
$$

Since $\nu_{A \otimes C}$ is a right $A$-linear map, so is $\lambda$. We first show that $\nu_{M}$ can be expressed in terms of $\lambda$. For any $M \in \mathbf{M}_{A}$ and $m \in M$ consider a morphism $\ell_{m}: A \otimes C \rightarrow M \otimes C$ in $\mathbf{M}_{A}^{C}(\psi)$ given by $a \otimes c \mapsto m \cdot a \otimes c$. Since the splitting of the adjunction $\Phi$ is natural in $\mathbf{M}_{A}^{C}(\psi)$ we have

$$
\ell_{m} \circ \nu_{A \otimes C}=\nu_{M \otimes C} \circ\left(\left(\ell_{m} \otimes_{A} \tilde{A}\right) \square_{\tilde{C}} C\right) .
$$

In particular, choosing $M=A$ one easily finds that (9) implies that $\nu_{A \otimes C}$ is a left $A$ module map. Now, if $M \in \mathbf{M}_{A}^{C}(\psi)$ one can take the morphism $\rho^{M} \in \operatorname{Hom}_{A}^{C}(M, M \otimes$ $C)$, and thus using the naturality of $\nu$, obtain $\rho^{M} \circ \nu_{M}=\nu_{M \otimes C} \circ\left(\rho^{M} \otimes C\right)$. In view of (9) this reads for all $\sum_{i} m_{i} \otimes \tilde{a}_{i} \otimes c_{i} \in(M \otimes \tilde{A}) \square_{\tilde{C}} C$ projected down to $\left(M \otimes_{A} \tilde{A}\right) \square_{\tilde{C}} C$

$$
\rho^{M} \circ \nu_{M}\left(\sum_{i} m_{i} \otimes \tilde{a}_{i} \otimes c_{i}\right)=\sum_{i} \ell_{m_{i(0)}} \circ \nu_{A \otimes C}\left(1_{A} \otimes m_{i(1)} \otimes \tilde{a}_{i} \otimes c_{i}\right) .
$$

Applying $M \otimes \epsilon_{C}$ to this last equality and using assumption that $(f, g)$ is admissible one obtains

$$
\nu_{M}\left(\sum_{i} m_{i} \otimes \tilde{a}_{i} \otimes c_{i}\right)=\sum_{i} m_{i(0)} \cdot \lambda\left(m_{i(1)} \otimes \tilde{a}_{i} \otimes c_{i}\right)
$$

In particular, the choice $M=A \otimes C$ gives for all $a \in A, \sum_{i} c_{i} \otimes \tilde{a}_{i} \otimes c_{i}^{\prime} \in(C \otimes \tilde{A}) \square_{\tilde{C}} C$

$$
\nu_{A \otimes C}\left(\sum_{i} a \otimes c_{i} \otimes \tilde{a}_{i} \otimes c_{i}^{\prime}\right)=a \sum_{i} \lambda\left(c_{i(2)} \otimes \tilde{a}_{i} \otimes c_{i}^{\prime}\right)_{\alpha} \otimes c_{i(1)}^{\alpha} .
$$

We are now ready to show that $\lambda$ satisfies all the conditions of Definition 3.2. Take any $x=\sum_{i} c_{i} \otimes \tilde{a}_{i} \otimes c_{i}^{\prime} \in(C \otimes \tilde{A}) \square_{\tilde{C}} C$, then

$$
\begin{aligned}
\sum_{i} \lambda\left(c_{i} \otimes \tilde{a}_{i} \otimes c_{i(1)}^{\prime}\right) \otimes c_{i(2)}^{\prime} & =\sum_{i}\left(A \otimes \epsilon_{C}\right) \circ \nu_{A \otimes C}\left(1_{A} \otimes c_{i} \otimes \tilde{a}_{i} \otimes c_{i(1)}^{\prime}\right) \otimes c_{i(2)}^{\prime} \\
& =\left(A \otimes \epsilon_{C} \otimes C\right) \circ\left(A \otimes \Delta_{C}\right) \circ \nu_{A \otimes C}\left(1_{A} \otimes x\right) \\
& =\nu_{A \otimes C}\left(1_{A} \otimes x\right) \\
& =\sum_{i} \lambda\left(c_{i(1)} \otimes \tilde{a}_{i} \otimes c_{i}^{\prime}\right)_{\alpha} \otimes c_{i(2)}^{\alpha} \quad(\text { by (10) }),
\end{aligned}
$$


where we used that $\nu_{A \otimes C}$ is a right $C$-comodule map to derive the second equality. This proves that $\lambda$ satisfies (4). Furthermore, for all $\sum_{i} c_{i} \otimes a_{i} \otimes \tilde{a}_{i} \otimes c_{i}^{\prime} \in(C \otimes A \otimes$ $\tilde{A}) \square{ }_{\tilde{C}} C$ we have

$$
\begin{aligned}
\lambda\left(\sum_{i} c_{i} \otimes f\left(a_{i}\right) \tilde{a}_{i} \otimes c_{i}^{\prime}\right) & =\left(A \otimes \epsilon_{C}\right) \circ \nu_{A \otimes C}\left(\sum_{i} 1_{A} \otimes c_{i} \otimes f\left(a_{i}\right) \tilde{a}_{i} \otimes c_{i}^{\prime}\right) \\
& =\left(A \otimes \epsilon_{C}\right) \circ \nu_{A \otimes C}\left(\sum_{i}\left(1_{A} \otimes c_{i}\right) \cdot a_{i} \otimes \tilde{a}_{i} \otimes c_{i}^{\prime}\right) \\
& =\left(A \otimes \epsilon_{C}\right) \circ \nu_{A \otimes C}\left(\sum_{i} a_{i \alpha} \otimes c_{i}^{\alpha} \otimes \tilde{a}_{i} \otimes c_{i}^{\prime}\right) \\
& =\sum_{i} a_{i \alpha} \lambda\left(c_{i}^{\alpha} \otimes \tilde{a}_{i} \otimes c_{i}^{\prime}\right),
\end{aligned}
$$

where we used the properties of the domain of $\nu_{A \otimes C}$ and the assumption that $-\square_{\tilde{C}} C$ preserves cokernel of $\mathrm{eq}_{M_{A} \tilde{A}}$ to derive the second equality. This proves that $\lambda$ satisfies (3). Finally, for all $c \in C, \Phi_{A \otimes C}\left(1_{A} \otimes c\right)=1_{A} \otimes c_{(1)} \otimes 1_{\tilde{A}} \otimes c_{(2)}$. Since $\nu_{A \otimes C}$ splits $\Phi_{A \otimes C}$ we have $1 \otimes c=\nu_{A \otimes C}\left(1_{A} \otimes c_{(1)} \otimes 1_{\tilde{A}} \otimes c_{(2)}\right)$. Applying $A \otimes \epsilon_{C}$ to this equality one immediately deduces that $\lambda$ satisfies (5). Therefore the morphism $(f, g)$ is totally integrable. This completes the proof of the first statement of the theorem.

(2) Given $\mathfrak{z}$ as in Definition 3.3 define for all $\tilde{M} \in \mathbf{M}_{A}^{C}(\tilde{\psi}), \nu_{\tilde{M}}: \tilde{M} \rightarrow\left(\tilde{M} \square_{\tilde{C}} C\right) \otimes_{A}$ $\tilde{A}, \nu_{\tilde{M}}=\left(\rho_{\tilde{M}} \otimes C \otimes_{A} \tilde{A}\right) \circ(\tilde{M} \otimes \mathfrak{z}) \circ \rho^{\tilde{M}}$. The proof that $\nu_{\tilde{M}}$ is the required cosplitting is dual to the proof of the corresponding part of assertion (1). Conversely, given a cosplitting $\nu_{\tilde{M}}$ define $\zeta=\left(\epsilon_{\tilde{C}} \otimes \tilde{A} \otimes C \otimes_{A} \tilde{A}\right) \circ \nu_{\tilde{C} \otimes \tilde{A}} \circ\left(\tilde{C} \otimes 1_{\tilde{A}}\right)$.

Notice that the assumption of Theorem 3.4(1) is satisfied if ${ }^{\tilde{C}} C$ is coflat. Dually, the assumption of Theorem 3.4 (2) is satisfied if ${ }_{A} \tilde{A}$ is flat. The remainder of the paper is devoted to the analysis of special cases of Theorem 3.4.

\section{Separable coalgebra-Galois extensions}

The following notion was introduced in [1]. It generalises the notion of an $H$-integral for a Doi-Hopf datum [6, Definition 2.1].

Definition 4.1. Let $(A, C)_{\psi}$ be an entwining structure. An integral in $(A, C)_{\psi}$ is an element $\mathfrak{z}=\sum_{i} a_{i} \otimes c_{i} \in A \otimes C$ such that for all $a \in A, a \cdot \mathfrak{z}=\mathfrak{z} \cdot a$. Explicitly, we require $\sum_{i} a a_{i} \otimes c_{i}=\sum_{i} a_{i} \psi\left(c_{i} \otimes a\right)$. An integral $\mathfrak{z}=\sum_{i} a_{i} \otimes c_{i}$ is said to be normalised if $\sum_{i} a_{i} \epsilon_{C}\left(c_{i}\right)=1$.

Example 4.2. Let $A$ be a Hopf algebra and $B \subset A$ be a left $A$-comodule subalgebra, i.e., a subalgebra of $A$ such that $\Delta_{A}(B) \subset A \otimes B$. Consider the coalgebra $C / B^{+} A$. $C$ is a right $A$-module in the natural way and there is an entwining structure $(A, C)_{\psi}$ with $\psi: c \otimes a \mapsto a_{(1)} \otimes c \cdot a_{(2)}$. Let $\Lambda \in C$ be such that for all $a \in A$, $\Lambda \cdot a=\epsilon_{A}(a) \Lambda$ and $\epsilon_{C}(\Lambda)=1$. Then $\mathfrak{z}=1 \otimes \Lambda$ is an integral in $(A, C)_{\psi}$.

Proof. Clearly, $1_{A} \epsilon(\Lambda)=1_{A}$. Take any $a \in A$, then $(1 \otimes \Lambda) \cdot a=a_{(1)} \otimes \Lambda \cdot a_{(2)}=$ $a \otimes \Lambda=a \cdot(1 \otimes \Lambda)$.

In [1] it has been shown that the existence of an integral in $(A, C)_{\psi}$ is closely related to the fact that the functor $-\otimes C: \mathbf{M}_{A} \rightarrow \mathbf{M}_{A}^{C}(\psi)$ of Example 2.7(1) is both left and right adjoint of the forgetful functor $\mathbf{M}_{A}^{C}(\psi) \rightarrow \mathbf{M}_{A}$. The following 
theorem, which is an entwining structure version of [5, Theorem 2.14], shows that integrals are closely related to the separability of $-\otimes C$.

Theorem 4.3. Let $(A, C)_{\psi}$ be an entwining structure. The functor $-\otimes C: \mathbf{M}_{A} \rightarrow$ $\mathbf{M}_{A}^{C}(\psi)$ is separable if and only if there exists a normalised integral in $(A, C)_{\psi}$.

Proof. Consider an admissible morphism $\left(A, \epsilon_{C}\right):(A, C)_{\psi} \rightarrow(A, k)_{\text {twist. }}$. Then $-\otimes C=-\square_{k} C: \mathbf{M}_{A} \rightarrow \mathbf{M}_{A}^{C}(\psi)$. Since ${ }_{A} A$ is flat, Theorem 3.4(2) can be applied and thus $-\otimes C$ is separable if and only if $\left(A, \epsilon_{C}\right)$ is totally cointegrable, i.e. there exists $\mathfrak{z} \in \operatorname{Hom}\left(k,(A \otimes C) \otimes_{A} A\right) \cong A \otimes C$ such that conditions (6) -(8) are satisfied. In this case condition (6) is empty, while condition (77) means that $\mathfrak{z}$ is an integral in $(A, C)_{\psi}$. Finally, condition (8) states that $\mathfrak{z}$ is normalised.

The existence of normalised integrals in the canonical entwining structure associated to a coalgebra-Galois extensions turns out to be equivalent to the separability of such an extension. First, recall from 13

Definition 4.4. An extension of algebras $B \hookrightarrow A$ is separable if there exists $u \in$ $A \otimes_{B} A$ such that for all $a \in A, a u=u a$ and $\mu_{A, B}(u)=1_{A}$, where $\mu_{A, B}: A \otimes_{B} A \rightarrow A$ is the natural map induced by $\mu_{A}$. The element $u$ is called a separability idempotent.

Proposition 4.5. A coalgebra-Galois extension $A(B)^{C}$ is separable if and only if there exists a normalised integral in the canonical entwining structure.

Proof. We first show that $\operatorname{can}^{-1}: A \otimes C \rightarrow A \otimes_{B} A$ is an $(A, A)$-bimodule map, where the $(A, A)$-bimodule structure on $A \otimes C$ is as in Definition 4.1. By construction, can $^{-1}$ is a left $A$-module map. For all $\mathfrak{z}=\sum_{i} a_{i} \otimes c_{i} \in A \otimes C, a \in A$

$$
\begin{aligned}
\operatorname{can}^{-1}(\mathfrak{z} \cdot a) & =\operatorname{can}^{-1}\left(\sum_{i} a_{i} a_{\alpha} \otimes c_{i}^{\alpha}\right)=\sum_{i} a_{i} \operatorname{can}^{-1}\left(a_{\alpha} \otimes c_{i}^{\alpha}\right) \\
& \left.=\sum_{i} a_{i} \operatorname{can}^{-1}\left(\operatorname{can}\left(\operatorname{can}^{-1}\left(1_{A} \otimes c_{i}\right) a\right)\right) \quad \text { (def. of canonical } \psi\right) \\
& =\sum_{i} a_{i} \operatorname{can}^{-1}\left(1_{A} \otimes c_{i}\right) a=\operatorname{can}^{-1}(\mathfrak{z}) a .
\end{aligned}
$$

Therefore $\mathfrak{z}$ is an integral in $(A, C)_{\psi}$ if and only if for all $a \in A$, au $=u a$, where $u=\operatorname{can}^{-1}(\mathfrak{z})$. Furthermore, directly from the definition of the canonical map can, one finds that $\left(A \otimes \epsilon_{C}\right) \circ$ can $=\mu_{A, B}$. Therefore $\mathfrak{z}$ is normalised if and only if $\mu_{A, B}(u)=1_{A}$.

Example 4.6. In the setting of Example 4.2, view $A$ as a right $C$-comodule via $\rho^{A}=(A \otimes \pi) \circ \Delta_{A}$, where $\pi: A \rightarrow C=A / B^{+} A$ is the canonical surjection, and assume that $B=\left\{b \in B \mid \forall a \in A, \rho^{A}(b a)=b \rho^{A}(a)\right\}$ (for example, this holds if either ${ }_{B} A$ or $A_{B}$ is faithfully flat). Then $A(B)^{C}$ is a coalgebra-Galois extension, and if there is $\Lambda \in C$ such that for all $a \in A, \Lambda \cdot a=\epsilon_{A}(a) \Lambda$ and $\epsilon_{C}(\Lambda)=1$, then $B \hookrightarrow A$ is separable.

The introduction of separable extensions in [13] was motivated by the Hochschild relative homological algebra [12]. In the case of a coalgebra-Galois extension the relationship between cohomology and separable extensions can be expressed in terms of integrals in the canonical entwining structure. Recall from [12 that if $B$ is 
a subalgebra of $A$ then for every $(A, A)$-bimodule $M$ the relative Hochschild cohomology groups $H^{n}(A, B, M)$ are defined as cohomology groups of the complex $\left(\bigoplus_{n=0}^{\infty} C^{n}(A, B, M), \delta\right)$, where $C^{0}(A, B, M)=\{m \in M \mid \forall b \in B, b \cdot m=m \cdot b\}$,

$$
C^{n}(A, B, M)={ }_{B} \operatorname{Hom}_{B}(\underbrace{A \otimes_{B} A \otimes_{B} \cdots \otimes_{B} A}_{n \text {-times }}, M), \quad n>0,
$$

and the coboundary $\delta: C^{n}(A, B, M) \rightarrow C^{n+1}(A, B, M)$ is given by

$$
\begin{aligned}
\delta(f)\left(a_{1}, \ldots, a_{n+1}\right)= & a_{1} \cdot f\left(a_{2}, \ldots a_{n+1}\right)+\sum_{i=1}^{n}(-1)^{i} f\left(a_{1}, \ldots, a_{i} a_{i+1}, \ldots, a_{n+1}\right) \\
& +(-1)^{n+1} f\left(a_{1}, \ldots, a_{n}\right) \cdot a_{n+1} .
\end{aligned}
$$

Corollary 4.7. Let $A(B)^{C}$ be a coalgebra-Galois extension. Then a normalised integral in the associated canonical entwining structure exists if and only if for all ( $A, A)$-bimodules $M, H^{1}(A, B, M)=0$.

Proof. By an argument similar to [9, p. 76], one shows, that the first relative Hochschild cohomology group is trivial for all $(A, A)$-bimodules if and only if the extension $B \hookrightarrow A$ is separable. Then the assertion follows from Proposition 4.5.

Corollary 4.8. Let $A(B)^{C}$ be a coalgebra-Galois extension with a normalised integral in the canonical entwining structure. Then any $(A, A)$-bimodule which is semisimple as a $(B, B)$-bimodule is semisimple as an $(A, A)$-bimodule.

Proof. By Corollary 4.7, for all $(A, A)$-bimodules $M, H^{1}(A, B, M)=0$. Then [12, Theorem 1] implies the assertion.

Dually one can consider

Definition 4.9. Let $(A, C, \psi)$ be an entwining structure. A $k$-module map $\mathfrak{y}$ : $C \otimes A \rightarrow k$, such that for all $a \in A, c \in C, c_{(1)} \mathfrak{y}\left(c_{(2)} \otimes a\right)=\mathfrak{y}\left(c_{(1)} \otimes a_{\alpha}\right) c_{(2)}{ }^{\alpha}$ is called a cointegral in $(A, C)_{\psi}$. A cointegral $\mathfrak{y}$ is said to be normalised if $\mathfrak{y} \circ\left(C \otimes 1_{A}\right)=\epsilon_{C}$.

Example 4.10. Let $C$ be a Hopf algebra and let $A$ be a right $C$-comodule algebra. Then $(A, C)_{\psi}$ is an entwining structure with $\psi: c \otimes a \mapsto a_{(0)} \otimes c a_{(1)}$. Let $\kappa \in A^{*}$ be such that $\kappa\left(1_{A}\right)=1$ and for all $a \in A, 1_{C} \kappa(a)=\kappa\left(a_{(0)}\right) a_{(1)}$. Then $\mathfrak{y}=\epsilon_{C} \otimes \kappa$ is a normalised cointegral in $(A, C)_{\psi}$.

Theorem 4.11. Let $(A, C)_{\psi}$ be an entwining structure. The functor $-\otimes A: \mathbf{M}^{C} \rightarrow$ $\mathbf{M}_{A}^{C}(\psi)$ is separable if and only if there exists a normalised cointegral in $(A, C)_{\psi}$.

Proof. Consider the morphism $\left(1_{A}, C\right):(k, C)_{\text {twist }} \rightarrow(A, C)_{\psi}$ and apply Theorem $3.4(1)$.

Definition 4.12. A coextension of coalgebras $C \rightarrow B$ is said to be a separable coextension if there exists a $k$-module map $v: C \square_{B} C \rightarrow k$ such that $(C \otimes v) \circ$ $\left(\Delta_{C} \otimes C\right)=(v \otimes C) \circ\left(C \otimes \Delta_{C}\right)$ on $C \square_{B} C$, and $v \circ \Delta_{C}=\epsilon_{C}$.

Proposition 4.13. An algebra-Galois coextension $C(B)_{A}$ is separable if and only if there exists a normalised cointegral in the associated canonical entwining structure. 
Example 4.14. Let $C$ be a Hopf algebra and $A \subset C$ a right comodule subalgebra of $C$, i.e., $\Delta_{C}(A) \subset A \otimes C$, so that we are in the setting of Example 4.10. Consider the coalgebra $B=C / C A^{+}$, and assume that $A=\left\{a \in C \mid \pi\left(a_{(1)}\right) \otimes a_{(2)}=\pi\left(1_{C}\right) \otimes a\right\}$, where $\pi: C \rightarrow B$ is the canonical surjection (this assumption is satisfied if either ${ }_{A} C$ or $C_{A}$ is faithfully flat). Then $C \rightarrow B$ is an $A$-Galois coextension and if there exists $\kappa \in A^{*}$ such that for all $a \in A, \kappa\left(a_{(1)}\right) a_{(2)}=\kappa(a) \epsilon_{C}$ and $\kappa\left(1_{A}\right)=1$, then this coextension is separable.

When $k=\mathbf{C}$, a rich source of separable coalgebra-Galois extensions is provided by quantum homogeneous spaces of compact quantum groups [21]. In this case we are in the setting of Example 4.14, with $C$ a compact quantum group and $A$ a right $C$-homogeneous quantum space. In many cases $C$ is a faithfully flat right or left $A$-module (see [16] for examples). The map $\kappa$ is the Haar measure on $C$ restricted to $A$. Perhaps the simplest example of this situation is when $C$ is the quantum $S U(2)$ group and $A$ is any of the quantum 2-spheres of Podleś [19].

\section{Split coalgebra-Galois extensions}

The following definition is a slightly modified version of [1, Definition 4.1]; both definitions describe the same object if a coalgebra $C$ is a finitely-generated projective $k$-module.

Definition 5.1. Let $(A, C)_{\psi}$ be an entwining structure. Any $\gamma \in \operatorname{Hom}(C \otimes C, A)$ such that the following diagrams

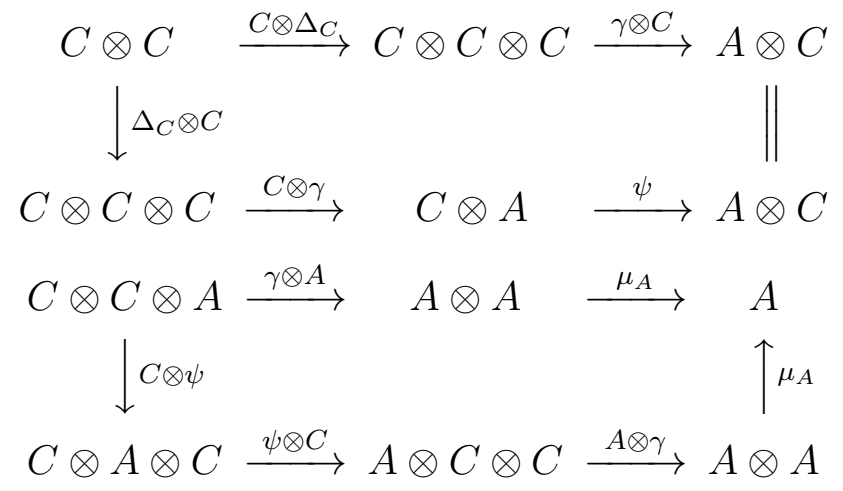

commute is called an integral map in $(A, C)_{\psi}$. An integral map $\gamma$ is said to be normalised, if for all $c \in C, \gamma\left(c_{(1)} \otimes c_{(2)}\right)=\epsilon_{C}(c) 1_{A}$.

The following theorem is an entwining structure version of [5, Theorem 2.3].

Theorem 5.2. The forgetful functor $\mathbf{M}_{A}^{C}(\psi) \rightarrow \mathbf{M}_{A}$ is separable if and only if there exists a normalised integral map in $(A, C)_{\psi}$.

Proof. Consider an admissible morphism $\left(A, \epsilon_{C}\right):(A, C)_{\psi} \rightarrow(A, k)_{\text {twist. }}$ Then $-\otimes_{A} A: \mathbf{M}_{A}^{C}(\psi) \rightarrow \mathbf{M}_{A}$ is the forgetful functor. In this case $(C \otimes A) \square_{k} C=C \otimes A \otimes C$, and for all $M \in \mathbf{M}_{A}$, $\mathrm{eq}_{M_{A} A}=M$ so that the assumption of Theorem 3.4(1) holds. Therefore the forgetful functor is separable if and only if $\left(A, \epsilon_{C}\right)$ is totally integrable, i.e., iff there exists $\lambda \in \operatorname{Hom}_{A}(C \otimes A \otimes C, A)$ satisfying all the conditions of Definition 3.2. Assume that such a $\lambda$ exists and define $\gamma=\lambda \circ\left(C \otimes 1_{A} \otimes C\right)$ : $C \otimes C \rightarrow A$. Then for all $a \in A, c, c^{\prime} \in C$ we have

$$
\begin{aligned}
a_{\alpha \beta} \gamma\left(c^{\beta} \otimes c^{\prime \alpha}\right) & \left.=a_{\alpha \beta} \lambda\left(c^{\beta} \otimes 1_{A} \otimes c^{\prime \alpha}\right)=\lambda\left(c \otimes a_{\alpha} \otimes c^{\prime \alpha}\right) \quad \text { (by (3) }\right) \\
& =\lambda\left(c \otimes 1_{A} \otimes c^{\prime}\right) a=\gamma\left(c \otimes c^{\prime}\right) a,
\end{aligned}
$$


where we used that $\lambda$ is a right $A$-module map to derive the penultimate equality. Hence the diagram (11) commutes. Also, (4) implies that the diagram (12) commutes, while the normalisation of $\gamma$ follows immediately from (5). Thus we conclude that $\gamma$ is a normalised integral map as required.

Conversely, assume that $\gamma$ is a normalised integral map and define $\lambda: C \otimes A \otimes C \rightarrow$ $A, c \otimes a \otimes c^{\prime} \mapsto a_{\alpha} \gamma\left(c^{\alpha} \otimes c^{\prime}\right)$. For all $a, a^{\prime} \in A, c, c^{\prime} \in C$ we have

$$
a_{\alpha} \lambda\left(c^{\alpha} \otimes a^{\prime} \otimes c^{\prime}\right)=a_{\alpha} a_{\beta}^{\prime} \gamma\left(c^{\alpha \beta} \otimes c^{\prime}\right)=\left(a a^{\prime}\right)_{\alpha} \gamma\left(c^{\alpha} \otimes c^{\prime}\right)=\lambda\left(c \otimes a a^{\prime} \otimes c^{\prime}\right),
$$

where (11) was used to obtain the third equality. This proves that the diagram (3) commutes. Furthermore

$$
\begin{array}{rlr}
\lambda\left(c_{(2)} \otimes a \otimes c^{\prime}\right)_{\alpha} \otimes c_{(1)}{ }^{\alpha} & =\left(a_{\delta} \gamma\left(c_{(2)} \delta \otimes c^{\prime}\right)\right)_{\alpha} \otimes c_{(1)}{ }^{\alpha} & \\
& =a_{\delta \alpha} \gamma\left(c_{(2)} \delta \otimes c^{\prime}\right)_{\beta} \otimes c_{(1)}{ }^{\alpha \beta} & \text { (by (1) ) } \\
& =a_{\alpha} \gamma\left(c^{\alpha}{ }_{(2)} \otimes c^{\prime}\right)_{\beta} \otimes c^{\alpha}{ }_{(1)}{ }^{\beta} & \text { (by (2) ) }) \\
& =a_{\alpha} \gamma\left(c^{\alpha} \otimes c^{\prime}{ }_{(1)}\right)_{\beta} \otimes c^{\prime}{ }_{(2)} & \text { (by (12) })) \\
& =\lambda\left(c \otimes a \otimes c^{\prime}{ }_{(1)}\right) \otimes c^{\prime}{ }_{(2)} . &
\end{array}
$$

This proves that diagram (41) commutes. Also,

$$
\begin{aligned}
\lambda\left(c \otimes a a_{\alpha}^{\prime} \otimes c^{\prime \alpha}\right) & \left.=\left(a a_{\alpha}^{\prime}\right)_{\beta} \gamma\left(c^{\beta} \otimes c^{\prime \alpha}\right)=a_{\beta} a_{\alpha \delta}^{\prime} \gamma\left(c^{\beta \delta} \otimes c^{\prime \alpha}\right) \quad \text { (by (11) }\right) \\
& =a_{\beta} \gamma\left(c^{\beta} \otimes c^{\prime}\right) a^{\prime}=\lambda\left(c \otimes a \otimes a^{\prime}\right)
\end{aligned}
$$

Therefore $\lambda$ is a right $A$-module map, and, consequently the morphism $\left(A, \epsilon_{C}\right)$ is integrable. The fact that it is totally integrable follows immediately from the normalisation of $\gamma$.

Example 5.3. Let $(A, C)_{\psi}$ be the canonical entwining structure associated to a pointed algebra-Galois coextension $C(k)_{A}$ of $k$. Then the forgetful functor $\mathbf{M}_{A}^{C}(\psi) \rightarrow$ $\mathbf{M}_{A}$ is separable.

Proof. Since $B=k, C \square_{B} C=C \otimes C$, and we define $\gamma=\left(\epsilon_{C} \otimes A\right) \circ \operatorname{cocan}^{-1}$ : $C \otimes C \rightarrow A$. We show that $\gamma$ is a normalised integral map. First notice that since $\operatorname{cocan}^{-1}$ is a left $C$-comodule map, one has $\operatorname{cocan}^{-1}=(C \otimes \gamma) \circ\left(\Delta_{C} \otimes C\right)$. Applying the definition of the canonical entwining map in Example 2.3 to cocan $^{-1}$ one immediately obtains $\psi \circ$ cocan $^{-1}=(\gamma \otimes C) \circ\left(C \otimes \Delta_{C}\right)$, i.e. $\psi \circ(C \otimes \gamma) \circ\left(\Delta_{C} \otimes C\right)=$ $(\gamma \otimes C) \circ\left(C \otimes \Delta_{C}\right)$. Thus we conclude that $\gamma$ satisfies condition (11]).

Let $\kappa: A \rightarrow k$ be the algebra map making $C(k)_{A}$ a pointed algebra-Galois coextension. One easily finds that $\rho_{C}=(\kappa \otimes C) \circ \psi$ and $C \otimes \kappa=\left(C \otimes \epsilon_{C}\right) \circ$ cocan. The map $\gamma$ is the cotranslation map, so, as explained in [3, Theorem 3.5], it has the following properties

$$
\begin{gathered}
\mu_{A} \circ(\gamma \otimes A)=\gamma \circ\left(C \otimes \rho_{C}\right), \\
\mu_{A} \circ(\gamma \otimes \gamma) \circ\left(C \otimes \Delta_{C} \otimes C\right)=\gamma \circ\left(C \otimes \epsilon_{C} \otimes C\right) .
\end{gathered}
$$


Using all these properties we obtain

$$
\begin{array}{rlr}
\mu_{A} \circ(\gamma \otimes A) & =\gamma \circ\left(C \otimes \rho_{C}\right) & \text { (by (13)) }) \\
& =\gamma \circ(C \otimes \kappa \otimes C) \circ(C \otimes \psi) \\
& =\gamma \circ\left(C \otimes \epsilon_{C} \otimes C\right) \circ(\operatorname{cocan} \otimes C) \circ(C \otimes \psi) \\
& =\mu_{A} \circ(\gamma \otimes \gamma) \circ\left(C \otimes \Delta_{C} \otimes C\right) \circ(\operatorname{cocan} \otimes C) \circ(C \otimes \psi) \quad \text { (by (14)) } \\
& =\mu_{A} \circ(A \otimes \gamma) \circ(\psi \otimes C) \circ(C \otimes \psi) & \text { (def. of } \psi) .
\end{array}
$$

This proves that $\gamma$ is an integral map. Finally, $\gamma$ is normalised by the normalisation property of the cotranslation map (cf. [3, Theorem 3.5]).

As explained in []] the separability of the forgetful functor implies various Maschketype theorems. Thus, similarly as in [1] we have

Corollary 5.4. If there is a normalised integral map in $(A, C)_{\psi}$, then

(1) Every object in $\mathbf{M}_{A}^{C}(\psi)$ which is semisimple as an object in $\mathbf{M}_{A}$ is semisimple as an object in $\mathbf{M}_{A}^{C}(\psi)$.

(2) Every object in $\mathbf{M}_{A}^{C}(\psi)$ which is projective (resp. injective) as a right $A$-module is a projective (resp. injective) object in $\mathbf{M}_{A}^{C}(\psi)$.

(3) $M \in \mathbf{M}_{A}^{C}(\psi)$ is projective as a right $A$-module if and only if there exists $V \in \mathbf{M}^{C}$ such that $M$ is a direct summand of $V \otimes A$ in $\mathbf{M}_{A}^{C}(\psi)(V \otimes A$ is an entwined module by Example 2.7(2)).

In the case of a coalgebra-Galois extension, the existence of normalised integral maps in the canonical entwining structure is closely related to the coalgebra-Galois extension being a split extension. Recall from [18] 14]

Definition 5.5. An extension of algebras $B \hookrightarrow A$ is called a split extension if there exists a unital $(B, B)$-bimodule map $E: A \rightarrow B$. The map $E$ is called a conditional expectation.

Proposition 5.6. A coalgebra-Galois extension $A(B)^{C}$ is a split extension if and only if there exists $\phi \in \operatorname{Hom}(C, A)$ such that

(i) $\forall c \in C, \quad \psi\left(c_{(1)} \otimes \phi\left(c_{(2)}\right)\right)=\phi(c) \rho^{A}\left(1_{A}\right)$,

(ii) $\sum_{i} a^{i} \phi\left(c_{i}\right)=1_{A}$, where $\sum_{i} a^{i} \otimes c_{i}=\rho^{A}\left(1_{A}\right)$.

(iii) $\forall b \in B, c \in C, \quad b_{\alpha} \phi\left(c^{\alpha}\right)=\phi(c) b$.

Proof. As explained in the proof of [2, Proposition 4.4], given a unital $(B, B)$ bimodule map $E: A \rightarrow B$ there exists $\phi \in \operatorname{Hom}(C, A)$ satisfying conditions (i)-(iii). Explicitly, $\phi=\left(A \otimes_{B} E\right) \circ \operatorname{can}^{-1} \circ\left(1_{A} \otimes C\right)$. Conversely, given $\phi \in \operatorname{Hom}(C, A)$ satisfying (i), [2, Theorem 4.3] implies that $E: A \rightarrow B, a \mapsto a_{(0)} \phi\left(a_{(1)}\right)$ is a left $B$-module map. Clearly, condition (ii) implies $E$ is unital. Furthermore, for all $a \in A, b \in B$

$$
E(a b)=(a b)_{(0)} \phi\left((a b)_{(1)}\right)=a_{(0)} b_{\alpha} \phi\left(a_{(1)}{ }^{\alpha}\right)=a_{(0)} \phi\left(a_{(1)}\right) b=E(a) b,
$$

where we used that $A \in \mathbf{M}_{A}^{C}(\psi)$ and the assumption (iii) to derive the second and third equalities respectively. This completes the proof.

Let $(A, C)_{\psi}$ be an entwining structure and assume that $A \in \mathbf{M}_{A}^{C}(\psi)$. Define $B$ as in Example 2.2. Then one can consider a covariant functor $(-)_{0}: \mathbf{M}_{A}^{C}(\psi) \rightarrow \mathbf{M}_{B}$

$$
M \mapsto M_{0}:=\left\{m \in M \mid \forall a \in A, \quad \rho^{M}(m \cdot a)=m \rho^{A}(a)\right\} .
$$


Notice, in particular, that $B=A_{0}$. As explained in [2] the functor $(-)_{0}$ is the right adjoint of the functor $-\otimes_{B} A: \mathbf{M}_{B} \rightarrow \mathbf{M}_{A}^{C}(\psi)$.

Corollary 5.7. If a coalgebra-Galois extension $A(B)^{C}$ is a split extension then ${ }_{B} A$ is a faithfully flat module. Consequently, the functors $-\otimes_{B} A: \mathbf{M}_{B} \rightarrow \mathbf{M}_{A}^{C}(\psi)$ and $(-)_{0}: \mathbf{M}_{A}^{C}(\psi) \rightarrow \mathbf{M}_{B}$ are inverse equivalences.

Proof. The first assertion follows from [2, Proposition 4.4], while the second is the consequence of [2, Corollary 3.11].

Proposition 5.8. Let $(A, C)_{\psi}$ be the canonical entwining structure associated to a coalgebra-Galois extension $A(B)^{C}$. If there is a normalised integral map in $(A, C)_{\psi}$ then $B \hookrightarrow A$ is a split extension.

Proof. Let $\gamma: C \otimes C \rightarrow A$ be a normalised integral map in $(A, C)_{\psi}$, and take $\phi: C \rightarrow A, c \mapsto \sum_{i} a_{\alpha}^{i} \gamma\left(c^{\alpha} \otimes c_{i}\right)$, where $\sum_{i} a^{i} \otimes c_{i}=\rho^{A}\left(1_{A}\right)$. Notice that the fact that $A$ is an $(A, C)_{\psi}$ module implies that for all $a \in A, \rho^{A}(a)=\sum_{i} a^{i} a_{\alpha} \otimes c_{i}^{\alpha}$. Furthermore, since $\rho^{A}$ is a coaction we have

$$
\sum_{i, j} a^{j} a_{\alpha}^{i} \otimes c_{j}^{\alpha} \otimes c_{i}=\sum_{i} a^{i} \otimes c_{i(1)} \otimes c_{i(2)} .
$$

We now show that $\phi$ satisfies all the conditions of Proposition 5.6. For all $c \in C$

$$
\begin{aligned}
& \psi\left(c_{(1)} \otimes \phi\left(c_{(2)}\right)\right)=\left(\sum_{i} a_{\alpha}^{i} \gamma\left(c_{(2)}^{\alpha} \otimes c_{i}\right)\right)_{\beta} \otimes c_{(1)}{ }^{\beta} \\
& \left.=\sum_{i} a_{\alpha \delta}^{i} \gamma\left(c_{(2)}^{\alpha} \otimes c_{i}\right)_{\beta} \otimes c_{(1)}^{\delta \beta} \quad \text { (by (1) }\right) \\
& \left.=\sum_{i} a_{\alpha}^{i} \gamma\left(c^{\alpha}{ }_{(2)} \otimes c_{i}\right)_{\beta} \otimes c^{\alpha}{ }_{(1)}{ }^{\beta} \quad \text { (by (2) }\right)
\end{aligned}
$$

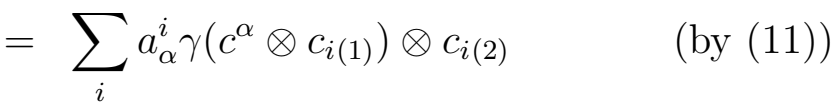

$$
\begin{aligned}
& =\sum_{i, j}\left(a^{j} a_{\beta}^{i}\right)_{\alpha} \gamma\left(c^{\alpha} \otimes c_{j}^{\beta}\right) \otimes c_{i} \quad \text { (by (15)) } \\
& \left.=\sum_{i, j} a_{\alpha}^{j} a_{\beta \delta}^{i} \gamma\left(c^{\alpha \delta} \otimes c_{j}^{\beta}\right) \otimes c_{i} \quad \text { (by (1) }\right) \\
& =\sum_{i, j} a_{\alpha}^{j} \gamma\left(c^{\alpha} \otimes c_{j}\right) a^{i} \otimes c_{i}=\phi(c) \rho^{A}\left(1_{A}\right) \quad \text { (by (12)) }
\end{aligned}
$$

Using normalisation of $\gamma$ as well as (15) one easily finds that $\sum_{i} a^{i} \phi\left(c_{i}\right)=1_{A}$. Finally, take any $b \in B, c \in C$ and compute

$$
\begin{aligned}
b_{\alpha} \phi\left(c^{\alpha}\right) & \left.=\sum_{i} b_{\alpha} a_{\beta}^{i} \gamma\left(c^{\alpha \beta} \otimes c_{i}\right)=\sum_{i}\left(b a^{i}\right)_{\alpha} \gamma\left(c^{\alpha} \otimes c_{i}\right) \quad \text { (by (1) }\right) \\
& =\sum_{i}\left(a^{i} b_{\beta}\right)_{\alpha} \gamma\left(c^{\alpha} \otimes c_{i}^{\beta}\right)=\sum_{i} a_{\alpha}^{i} b_{\beta \delta} \gamma\left(c^{\alpha \delta} \otimes c_{i}^{\beta}\right) \quad(b \in B,(1)) \\
& \left.=\sum_{i} a_{\alpha}^{i} \gamma\left(c^{\alpha} \otimes c_{i}\right) b=\phi(c) b \quad \text { (by (12) }\right)
\end{aligned}
$$

Therefore $\phi$ satisfies all the conditions of Proposition 5.6 and, consequently, $B \hookrightarrow A$ is a split extension. 
Dually to Definition 5.1 we can consider

Definition 5.9. Let $(A, C)_{\psi}$ be an entwining structure. Any $\zeta \in \operatorname{Hom}(C, A \otimes A)$ such that the following diagrams

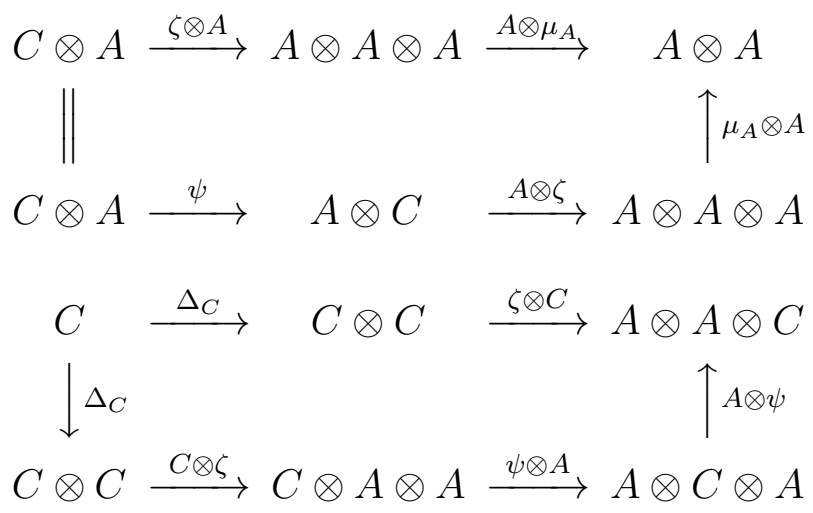

commute is called a cointegral map in $(A, C)_{\psi}$. A cointegral map $\zeta$ is said to be normalised, if $\mu_{A} \circ \zeta=1_{A} \circ \epsilon_{C}$.

Theorem 5.10. The forgetful functor $\mathbf{M}_{A}^{C}(\psi) \rightarrow \mathbf{M}^{C}$ is separable if and only if there exists a normalised cointegral map in $(A, C)_{\psi}$.

Proof. Consider an admissible morphism $\left(1_{A}, C\right):(k, C)_{\sigma} \rightarrow(A, C)_{\psi}$ and apply Theorem 3.4 (2).

Example 5.11. Let $(A, C)_{\psi}$ be a canonical entwining structure associated to a copointed coalgebra-Galois extension $A(k)^{B}$ of $k$. Then the forgetful functor $\mathbf{M}_{A}^{C}(\psi) \rightarrow$ $\mathbf{M}^{C}$ is separable.

In this case a normalised cointegral map is $\zeta=\operatorname{can}^{-1} \circ\left(1_{A} \otimes C\right)$.

\section{Strongly Separable CoAlgebra-Galois extensions}

In this section we combine the results of previous two sections to determine when a coalgebra-Galois extension is a strongly separable extension. Such an extension was introduced in [14] in order to describe algebraic aspects of the Jones knot polynomial.

Definition 6.1. An extension of algebras $B \hookrightarrow A$ is called a strongly separable extension if it is a separable and split extension, and there exist a separation idempotent $u=\sum_{i} u_{i} \otimes u^{i}$, a conditional expectation $E: A \rightarrow B$ and a unit $\tau \in k$ such that for all $a \in A$,

(i) $\sum_{i} E\left(a u_{i}\right) u^{i}=a \tau$

(ii) $\sum_{i} u_{i} E\left(u^{i} a\right)=a \tau$.

Proposition 6.2. Let $A(B)^{C}$ be a coalgebra-Galois extension. If there exist a normalised integral $\mathfrak{z}=\sum_{i} a_{i} \otimes c_{i}$ and a normalised integral map $\gamma \in \operatorname{Hom}(C \otimes C, A)$ in the canonical entwining structure $(A, C)_{\psi}$, and a unit $\tau \in k$ such that

(i) $\sum_{i} a_{i} 1_{A(0)} \gamma\left(c_{i}^{\alpha} \otimes 1_{A(1)}\right)=\tau$,

(ii) $\sum_{i} a_{i(0)} \gamma\left(a_{i(1)} \otimes c_{i}\right)=\tau$,

then $B \hookrightarrow A$ is a strongly separable extension. 
Proof. By Proposition 4.5, $B \hookrightarrow A$ is separable with $u=\sum_{i} u_{i} \otimes u^{i}=\operatorname{can}^{-1}(\mathfrak{z})$, while by Proposition 5.8, $B \hookrightarrow A$ is split with a conditional expectation $E: A \mapsto$ $a_{(0)} 1_{A(0)} \gamma\left(a_{(1)}^{\alpha} \otimes 1_{A(1)}\right)=\left(a 1_{A(0)}\right)_{(0)} \gamma\left(\left(a 1_{A(0)}\right)_{(1)} \otimes 1_{A(1)}\right)$. Take any $a \in A$ and compute:

$$
\begin{aligned}
& \sum_{i} E\left(a u_{i}\right) u^{i}=\sum_{i} E\left(u_{i}\right) u^{i} a \quad(u \text { is an integral }) \\
& =\sum_{i} u_{i(0)} 1_{A(0)} \gamma\left(u_{i(1)}^{\alpha} \otimes 1_{A(1)}\right) u^{i} a \\
& =\sum_{i} u_{i(0)} 1_{A(0) \alpha} u_{\beta \delta}^{i} \gamma\left(u_{i(1)}^{\alpha \delta} \otimes 1_{A(1)}^{\beta}\right) a \quad \text { (by (12)) } \\
& \left.=\sum_{i} u_{i(0)}\left(1_{A(0)} u_{\beta}^{i}\right)_{\alpha} \gamma\left(u_{i(1)}^{\alpha} \otimes 1_{A(1)}^{\beta}\right) a \quad \text { (by (田) }\right) \\
& =\sum_{i} u_{i(0)} u_{(0) \alpha}^{i} \gamma\left(u_{i(1)}^{\alpha} \otimes u_{(1)}^{i}\right) a \quad\left(A \in \mathbf{M}_{A}^{C}(\psi)\right) \\
& =\sum_{i}\left(u_{i} u_{(0)}^{i}\right)_{(0)} \gamma\left(\left(u_{i} u_{(0)}^{i}\right)_{(1)} \otimes u_{(1)}^{i}\right) a \quad\left(A \in \mathbf{M}_{A}^{C}(\psi)\right) \\
& =\sum_{i} a_{i(0)} \gamma\left(a_{i(1)} \otimes c_{i}\right) a=\tau a \quad(\mathfrak{z}=\operatorname{can}(u))
\end{aligned}
$$

Therefore the condition Definition 6.1(i) is satisfied. Furthermore

$$
\begin{aligned}
\sum_{i} u_{i} E\left(u^{i} a\right) & =\sum_{i} a u_{i} E\left(u^{i}\right) \quad(u \text { is an integral }) \\
& =\sum_{i} a u_{i} u_{(0)}^{i} 1_{A(0)_{\alpha}} \gamma\left(u_{(1)}^{i} \otimes 1_{A(1)}\right) \\
& =\sum_{i} a a_{i} 1_{A(0)_{\alpha}} \gamma\left(c_{i}^{\alpha} \otimes 1_{A(1)}\right)=\tau a \quad(\mathfrak{z}=\operatorname{can}(u))
\end{aligned}
$$

This proves Definition 6.1(ii) and thus completes the proof of the proposition.

Proposition 6.3. Let $k$ be a field and let $A(B)^{C}$ be a coalgebra-Galois extension with both $A$ and $B$ finite dimensional. Suppose that $A_{B}$ is free. Then $B \hookrightarrow A$ is a strongly separable extension if and only if there exists a normalised integral $\mathfrak{z}=\sum_{i} a_{i} \otimes c_{i}$ in the canonical entwining structure $(A, C)_{\psi}$, a map $\phi: C \rightarrow A$ satisfying conditions (i)-(iii) in Proposition 5.6, and a non-zero $\tau \in k$ such that

$$
\sum_{i} a_{i} \phi\left(c_{i}\right)=\tau
$$

Proof. By Proposition 4.5, $B \hookrightarrow A$ is separable with $u=\sum_{i} u_{i} \otimes u^{i}=\operatorname{can}^{-1}(\mathfrak{z})$, while by Proposition 5.6, $B \hookrightarrow A$ is split with a conditional expectation $E: a \mapsto$ $a_{(0)} \phi\left(a_{(1)}\right)$. By [11, Remark 1.4(d)], Definition 6.1(i) holds provided that condition Definition 6.1(ii) holds. Thus it suffices to prove that (18) is a sufficient and 
necessary condition for Definition 6.1(ii). Take any $a \in A$ and compute:

$$
\begin{aligned}
\sum_{i} u_{i} E\left(u^{i} a\right) & =\sum_{i} a u_{i} E\left(u^{i}\right) \quad(u \text { is an integral }) \\
& =\sum_{i} a u_{i} u_{(0)}^{i} \phi\left(u_{(1)}^{i}\right)=\sum_{i} a a_{i} \phi\left(c_{i}\right) .
\end{aligned}
$$

Therefore $\sum_{i} u_{i} E\left(u^{i} a\right)=\tau a$ if and only if (18) holds.

\section{REFERENCES}

[1] T. Brzeziński. Frobenius properties and Maschke-type theorems for entwined modules. Preprint, math/9806025, 1998. To appear in Proc. Amer. Math. Soc.

[2] T. Brzeziński. On modules associated to coalgebra-Galois extensions. Preprint, $\mathrm{q}-$ alg/9712023, 1997. To appear in J. Algebra.

[3] T. Brzeziński and P.M. Hajac. Coalgebra extensions and algebra coextensions of Galois type. Preprint, q-alg/9708010, 1997. To appear in Commun. Algebra.

[4] T. Brzeziński and S. Majid. Coalgebra bundles. Commun. Math. Phys., 191:467492, 1998.

[5] S. Caeneepel, B. Ion, G. Militaru and S. Zhu. Separable functors for the category of Doi-Hopf modules. Applications. Adv. Math. to appear.

[6] S. Caeneepel, G. Militaru and S. Zhu. Doi-Hopf modules, Yetter-Drinfel'd modules and Frobenius type properties. Trans. Amer. Math. Soc. , 349:4311-4342, 1997.

[7] S. Caeneepel and S. Raianu. Induction functors for the Doi-Koppinen unified Hopf modules. [in:] Abelian Groups and Modules, A. Facchini and C. Menini (eds.), Kluwer Academic Publishers, Dordrecht 1995, pp. 73-94.

[8] A. del Rio. Categorical methods in graded ring theory. Publ. Mat., 36:489-531, 1992.

[9] F. DeMeyer and E. Ingraham. Separable Algebras Over Commutative Rings. Lecture Notes in Mathematics 181, Springer-Verlag, Berlin/Heidelberg/New York, 1971.

[10] Y. Doi. Unifying Hopf modules. J. Algebra, 153:373-385, 1992.

[11] D. Fischman, S. Montgomery and H.-J. Schneider Frobenius extensions of subalgebras of Hopf algebras. Trans. Amer. Math. Soc. 349:4857-4895, 1997.

[12] G. Hochschild. Relative homological algebra. Trans. Amer. Math. Soc. 82:246-269, 1956.

[13] K. Hirata and K. Sugano. On semisimple extensions and separable extensions over non commutative rings. J. Math. Soc. Japan 18:360-374, 1966.

[14] L. Kadison. The Jones polynomial and certain separable Frobenius extensions. $J$. Algebra 186:461-475, 1996.

[15] M. Koppinen. Variations on the smash product with applications to group-graded rings. J. Pure Appl. Alg. , 104:61-80, 1994.

[16] E.F. Müller and H.-J. Schneider. Quantum homogeneous spaces with faithfully flat module structure. Preprint, 1998.

[17] C. Năstăsescu, M. van den Bergh and F. van Oystaeyen. Separable functors applied to graded rings. J. Algebra 123:397-413, 1989.

[18] R. Pierce. Associative algebras. Springer-Verlag, New York/Berlin, 1982.

[19] P. Podleś. Quantum spheres. Lett. Math. Phys., 14:193-202, 1987.

[20] M.D. Rafael. Separable functors revisited. Commun. Algebra 18:1445-1459, 1990.

[21] S.L. Woronowicz. Compact matrix pseudogroups. Commun. Math. Phys., 111:613$665,1987$.

Department of Mathematics, University of York, Heslington, York YO10 5DD, U.K.

E-mail address: tb10@york.ac.uk

$U R L:$ http//www.york.ac.uk/ tb10 